\title{
GRANULOMA ANULAR PAPULAR UMBILICADO EN UN ADOLESCENTE
}

\author{
María Ximena Tobón ${ }^{1}$ Állvaro Tovar ${ }^{2}$, Gerzaín Rodríguez ${ }^{3}$.
}

${ }^{1}$ Residente de Dermatología, Universidad CES, Centro Dermatológico Federico Lleras Acosta E.S.E. Bogotá, Colombia.

${ }^{2}$ Dermatólogo, Centro Dermatológico Federico Lleras Acosta E.S.E. Bogotá, Colombia.

${ }^{3}$ Dermatopatólogo consultor, Centro Dermatológico Federico Lleras Acosta E.S.E. Bogotá, Colombia.

Recibido: Septiembre 10 de 2012 Aceptado: Septiembre 27 de 2012

\section{Resumen}

El granuloma anular es una enfermedad granulomatosa benigna con diferentes manifestaciones clínicas que incluye la forma localizada, generalizada, perforante, subcutánea, en parche, papular y lineal. El granuloma anular papular umbilicado es considerado una variante de la forma papular y en la literatura anglosajona se han reportado muy pocos casos. Se presenta el caso de un adolescente con pápulas eucrómicas asintomáticas en el dorso de las manos desde la infancia. La biopsia sugirió liquen nitidus, posteriormente la correlación clínico-patológica permitió hacer diagnóstico de granuloma anular papular umbilicado. Se resalta la importancia de este diagnóstico diferencial y la cronicidad de las lesiones.

Palabras clave: Granuloma anular, papular, umbilicado, liquen nitidus, correlación clínico-patológica.

\section{PAPULAR UMBILICATED GRANULOMA ANNULARE IN AN ADOLESCENT}

\begin{abstract}
The granuloma annulare is a benign granulomatous disease with different clinical manifestations including, localized, generalized, perforating, subcutaneous, patch type, papular and linear types. Papular umbilicated granuloma annulare is considered to be a variant of the papular type and in the Anglo-Saxon literature very few cases have been reported.

We describe the case of an adolescent with asymptomatic skin colored papules on the dorsal aspect of the hands since infancy. The biopsy suggested lichen nitidus and after the clinicopathological correlation the diagnosis of papular umbilicated granuloma annulare was made. We highlight the importance of this differential diagnosis and the chronicity of the lesions.
\end{abstract}

Key words: Papular, umbilicated, granuloma annulare, lichen nitidus, clinicopathological correlation.

\section{GRANULOMA ANULAR PAPULAR UMBILICADO EM UM ADOLESCENTE}

\begin{abstract}
Resumo
O granuloma anular é uma doença granulomatosa benigna com diferentes manifestações clínicas que inclui a forma localizada, generalizada, perfurante, subcutânea, em parche, papular e linear. O granuloma anular papular umbilicado é considerado uma variante da forma papular e na literatura anglo-saxã foram relatados muito poucos casos. Apresenta-se o caso de um adolescente com pápulas acrômicas assintomáticas no dorso das mãos desde a infância. A biopsia sugeriu líquen nitidus, posteriormente a correlação clínico-patológica permitiu fazer diagnóstico de granuloma anular papular umbilicado. Destaca-se a importância deste diagnóstico diferencial e a cronicidade das lesões.
\end{abstract}

Palavras chave: Granuloma anular, papular, umbilicado, líquen nitidus, correlação clínico-patológica.

* Correspondencia: María Ximena Tobón Montes de Oca Avenida 1a Nº 13 A - 61 Centro Dermatológico Federico Lleras Acosta E.S.E. Teléfono: (571) 2428160 - Fax: 3373597-2899724 mariatobon@hotmail.com 


\section{Introducción}

El granuloma anular (GA) es una dermatosis granulomatosa benigna frecuente en niños y adolescentes (1), con diferentes manifestaciones clínicas incluyendo la forma localizada, generalizada, perforante, subcutánea, en parche, papular y lineal. El GA papular umbilicado fue descrito por primera vez por Lucky et $\mathrm{al}^{2}$. Se piensa que la umbilicación central puede ser el resultado de la degeneración focal de colágeno, sin embargo, en la biopsia no suele encontrarse una perforación del tejido conectivo degenerado. Por este motivo se ha propuesto que es la forma intermedia entre el GA papular y el GA perforante. Se han reportado muy pocos casos de esta entidad; en 2011 Eujin et al. describen que solo se habían reportado 6 casos en la literatura anglosajona y reportan dos más con presentaciones inusuales (2).

\section{Caso clínico}

Adolescente de 14 años de edad quien presenta al examen físico pápulas eucrómicas asintomáticas de 2 a $3 \mathrm{~mm}$ de diámetro, firmes, agrupadas, con umbilicación y descamación central, localizadas en el dorso de las manos (Figuras 1 y 2) desde el primer año de vida, sin evidenciar cambios desde su aparición. Se hizo una impresión diagnóstica clínica de granuloma anular perforante.

La histología de una de estas pápulas reportó piel gruesa con un infiltrado papilar constituido por histiocitos incluidos en colágeno denso acompañado de pocos linfocitos que comprime y atrofia la epidermis, limitado por repliegues de la misma y que ensancha la dermis papilar (Figura 3).

Los hallazgos histológicos observados fueron interpretados inicialmente como liquen nitidus, pero la correlación clínico-patológica permitió hacer diagnóstico de granuloma anular papular umbilicado. Se inició tratamiento con clobetasol tópico.

\section{Discusión}

El granuloma anular (GA) es una dermatosis frecuente en niños y adolescentes (1), con una presentación clínica variable dentro de la que se incluyen las formas localizada, diseminada, subcutánea, perforante, en parche, papular y lineal (2). La variante perforante se caracteriza por la presencia de pápulas eucrómicas umbilicadas que representan la eliminación transepi-

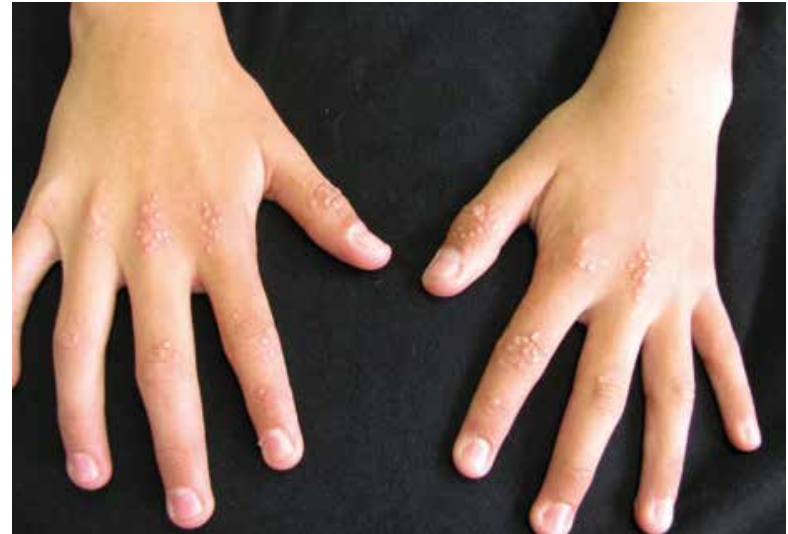

Figura 1. Aspecto de las pápulas en el dorso de las manos.

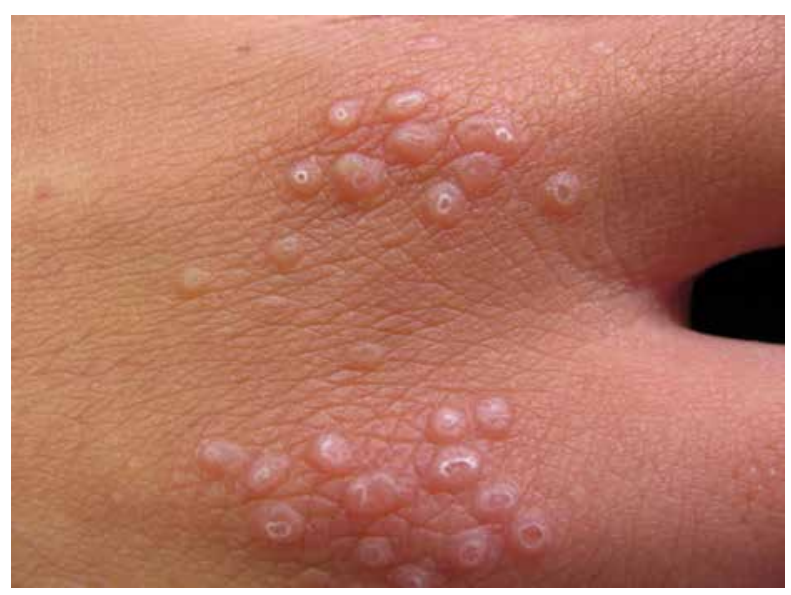

Figura 2. Pápulas con umbilicación y descamación central.

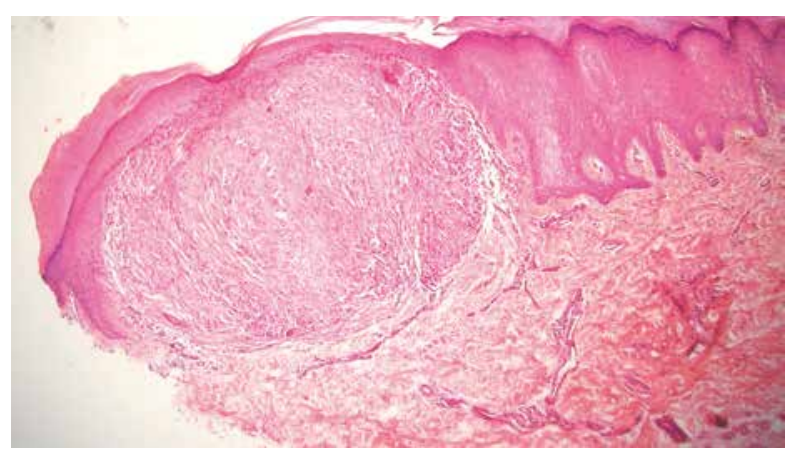

Figura 3. Infiltrado en la dermis papilar.

dérmica del colágeno necrobiótico. Se han descrito formas clínicas papulares umbilicadas mal definidas, que según algunos autores representan un subtipo de la variante perforante, en las cuales la histología se 
confunde con la de otras enfermedades inflamatorias (3), mientras que otros lo consideran una variante de la forma papular (2).

El aspecto de estas lesiones, su localización, evolución, edad de aparición, tendencia a la cronicidad y dificultad diagnóstica son algunas de las características que se han descrito en casos denominados granuloma anular papular umbilicado, entidad de difícil diagnóstico debido a su presentación clínica e histológica (4). En este paciente la correlación clínica sugirió la posibilidad de granuloma anular papular umbilicado, diagnóstico compatible con los hallazgos histológicos reportados. El GA papular umbilicado fue descrito por primera vez por Lucky et al. Se piensa que la umbilicación central puede ser el resultado de la degeneración focal de colágeno, sin embargo, esto en la biopsia no suele encontrarse una perforación del tejido conectivo degenerado. Por este motivo se ha propuesto que el GA papular umbilicado es la forma intermedia entre el GA papular y el GA perforante (2).

Teniendo en cuenta que la imagen en la panorámica a bajo aumento se interpretó inicialmente como liquen nitidus, es importante establecer las similitudes y diferencias entre estas dos entidades. Las lesiones típicas de liquen nitidus consisten en múltiples y diminutas pápulas eucrómicas y brillantes que confluyen, de predominio en la superficie flexora de los miembros superiores, genitales y tronco anterior (5); mientras que la presentación característica del GA papular umbilicado son pápulas asintomáticas, firmes, color piel, con umbilicación central, localizadas en el dorso de las manos (4). Esta variedad puede representar hasta un $5 \%$ de todos los casos de GA y comúnmente afecta niños y adultos jóvenes al igual que el liquen nitidus $(1,5)$, lo cual coincide con la clínica y edad de aparición en el paciente que se presenta. A pesar de que ambas entidades suelen ser autolimitadas, el GA papular umbilicado suele persistir durante años (1), como sucedió en este caso, mientras que el liquen nitidus rara vez persiste indefinidamente (3).

Los diagnósticos diferenciales clínicos, en este caso, incluyen molusco contagioso, liquen nitidus, sarcoidosis papular y verrugas planas (2).
En la histopatología del GA papular umbilicado se observan granulomas en empalizada con colágeno denso y linfocitos, sin células epitelioides, con adelgazamiento de la epidermis suprayacente (1) y paraqueratosis, sin una perforación real (4), hallazgos compatibles con la biopsia de este paciente; mientras que en el liquen nitidus predomina el infiltrado de linfocitos y células epitelioides inmediatamente por debajo de la epidermis, asociado a elongación de las crestas interpapilares en "garra" y adelgazamiento de la epidermis suprayacente, puede haber paraqueratosis sin hipergranulosis $(5,6)$. Es importante realizar cortes seriados del bloque, ya que en caso de existir una verdadera perforación con eliminación transepidérmica de colágeno degenerado, ésta podría no verse y dificultaría el diagnóstico (4).

\section{Conclusión}

El granuloma anular papular umbilicado es una variante del GA perforante de difícil diagnóstico, ya que no presenta las características clínicas e histológicas usuales del GA (4).

Es de gran importancia conocer la clínica y la histopatología de estas dos entidades, para lograr una aproximación diagnóstica adecuada. El aspecto morfológico con la depresión central es muy sugestivo de GA y permitió hacer la correlación clínico-patológica de una variedad poco frecuente de GA.

\section{REFERENCIAS}

1. Batchelor R, Clark S. Clearance of generalized papular umbilicated granuloma annulare in a child with bath PUVA therapy. Pediatr Dermatol. 2006; 23: 72-4.

2. Cho E, Cho SH, Lee JD. Unusual presentations of papular umbilicated granuloma annulare. J Dermatol. 2011; 38(4): 402-4.

3. Prendiville J. Granuloma annulare. En: Wolff K, Goldsmith L, Katz S, Gilchrest B, Paller A, Leffell D.. New York: McGraw-Hill; 2008. p. 369-73.

4. Lucky A, Prose N, Bove K, White W, Jorizzo J. Papular umbilicated granuloma annulare, A report of four pediatric cases. Arch Dermatol. 1992; 128: 1375-8.

5. Shiohara T, Kano Y. Lichen planus and lichenoid dermatoses. En: Bolognia JL, Jorizzo JL, Rapini RP. Dermatology. España: Elsevier; 2008. p 159-80.

6. Pittelkow MR, Daoud MS. Lichen nitidus. En: Wolff K, Goldsmith L, KatzS, Gilchrest B, Paller A, Leffell D. Fitzpatrick's Dermatology in General Medicine. New York: McGraw-Hill; 2008. p. 255-8. 